\title{
On the criteria for strong and weak polarization responses of ordered objects and systems
}

\author{
Yakov Fofanov ${ }^{1, *}$, Igor Sokolov ${ }^{2}$, Ivan Pleshakov ${ }^{2,3}$, Vasiliy Vetrov ${ }^{4}$, Andrey Prokofiev ${ }^{2,3}$, \\ Aleksey Kuraptsev ${ }^{3}$, and Efim Bibik ${ }^{5}$ \\ ${ }^{1}$ Institute for Analytical Instrumentation RAS, 190103, St. Petersburg, Russia \\ ${ }^{2}$ Ioffe Physical Technical Institute RAS, 194021, St. Petersburg, Russia \\ ${ }^{3}$ Peter the Great St. Petersburg Polytechnic University, 195251, St. Petersburg, Russia \\ ${ }^{4}$ S. I. Vavilov State Optical Institute, 199034, St. Petersburg, Russia \\ ${ }^{5}$ St. Petersburg State Technological Institute, 190013, St. Petersburg, Russia
}

\begin{abstract}
The fundamental system of criteria for strong and weak polarization responses of ordered objects and systems is considered. It is shown that the weak responses have important properties of linearity (proportionality) and additivity. As an example, the responses of magnetically ordered materials, optical crystals and ensembles of magnetic nanoparticles are considered. When scanning laser probing of the modified leucosapphire crystals, oscillations of weak polarization responses in laser radiation scattered on texture of crystals are recorded. Investigations of magnetic fluids polarization responses have shown their high sensitivity to introduction of coagulating impurities and to processes of agglomeration of magnetic nanoparticles.
\end{abstract}

The prospects for the further development and application of high sensitive methods for laser polarization-optical probing of matter may be limited in a number of cases by the lack of sufficiently complete ideas about their capabilities. Here some aspects of quantitative analysis of polarization responses for objects and systems with small optical anisotropy are considered $[1,2]$.

A natural measure of the smallness of the observed optical anisotropy $\Delta$ can be the inequality $\Delta<<\Delta_{\mathrm{LSL}} \sim 1 \mathrm{rad}$ (here LSL - linearity scale limit). It is possible to call the studied objects (samples) meeting this criterion the samples with "weak anisotropy" or "weak samples", and the polarization responses corresponding to them (signals in the optoelectronic channel) - "weak responses" (or "weak signals"). In this case the observed responses have important properties of linearity (proportionality) and additivity.

Two other criteria take into account the photon (shot) noise of probing light, that considered as an instrument for researches (shot noise limit - SNL), and thermal noise of the registration system (thermal noise limit - TNL). To ensure that these noises do not affect the results of observations, polarization responses must be strong in according to both criteria: $\Delta$ $>\Delta_{\mathrm{SNL}}$ and $\Delta>\Delta_{\mathrm{TNL}}$. In accordance with these criteria, the region $\Delta<<\Delta_{\mathrm{SNL}}$ may naturally be called the region of superweak (or superlow) signals - SWS [3-6].

"Corresponding author: yakinvest@yandex.ru 
The additivity of weak polarization responses allows the use of a scalar approximation (scalar model), which does not take into account the polarization of light. However, this can lead to incorrect results and in the general case only the vector model should be used [7].

Thus, when the objects under study are probed by laser radiation with a strong polarization modulation, there is a very wide (about to seven orders of magnitude) and a very convenient range of measured responses from about $\Delta_{\mathrm{SNL}} \sim 10^{-4}$ ang. min. up to values of the order of $\Delta_{\mathrm{LSL}} \sim 10^{3} \mathrm{ang}$. min. The examples of objects which weak polarization responses characterize the processes of structure formation, ordering, agglomeration, etc. happening in them are further considered.

It was found that polarization magneto-optical responses of model magnetic fluid (magnetite nanoparticles in kerosene) are proportional to volume concentration of solidphase in the range which is about four orders $\left(10^{-2}-10^{-6}\right)$, [8]. Fig. 1 illustrates the qualitative change of the polarization response of the investigated fluid in the case of addition of the coagulating impurity (an excess amount of surfactant).

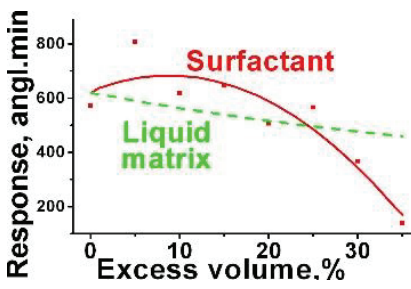

Fig. 1. Excess of surfactant.

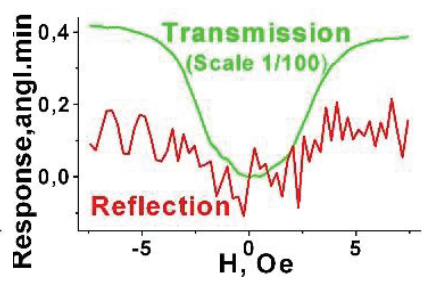

Fig. 2. Response of iron borate.

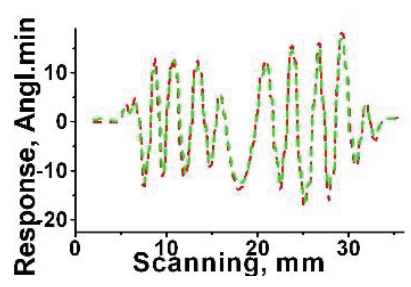

Fig. 3. Scattering in polar. lens

Polarization magneto-optical responses of an iron borate crystal at observation on transmitted and reflected light are presented in Fig. 2. It is seen that in the region of unsaturated magnetic fields the characteristic quadratic dependence of observed weak responses on the field is observed (Kotton-Mouton effect) [9].

Fig. 3 shows the oscillations of a weak polarization response in laser radiation scattered by the polarization lens material (leucosapphire). These oscillations indicate existence of textural heterogeneity and the formation of strong polarization structure due to the modification (bending) of the original crystal of leucosapphire [10].

This study was carried out with the financial support of the Russian Foundation for Basic Research (Grant No. 15-02-08703 a).

\section{References}

[1] Ya.A. Fofanov, Proceedings SPIE 1811, 413 (1992)

[2] I.M. Sokolov, Ya.A. Fofanov, J. Opt. Soc. Am. A 12, 1579 (1995)

[3] Ya.A. Fofanov, Advances in Optoelectronics Research (Nova Science Publishers, Inc., USA, 2014), 75-114

[4] Y.A. Fofanov, Proceedings SPIE 7993, 79930 O (2011)

[5] A.V. Belinsky, D.V. Volkov, A.V. Dmitriev, M.Kh. Shulman, JETP 117, 771 (2013)

[6] A.V. Belinsky, M.Kh. Shulman, Physics-Uspekhi 57, 1022 (2014)

[7] Ya.A. Fofanov, A.S. Kuraptsev, I.M. Sokolov, M.D. Havey, Phys. Rev. A 84, 053811 (2011)

[8] Ya.A. Fofanov et al., Theor. Phys. Lett. 42, 1054 (2016)

[9] Ya.A. Fofanov, I.V. Pleshakov, Yu.I. Kuz'min, J. Opt. Technol. 80, 64 (2013)

[10] V.N. Vetrov, B.A. Ignatenkov, Crystallography Reports 58, 474 (2013) 